Supporting Information

\title{
Photoredox Iridium-Nickel Dual Catalyzed Cross-Electrophile Coupling: From Batch to Continuous Stirred-Tank Reactor via Automated Segmented Flow Reactor
}

Rohit Duvadie, ${ }^{\# 1}$ Alexander Pomberger ${ }^{\#},{ }^{2}$ Yiming Mo, ${ }^{2}$ Erhan I. Altinoglu, ${ }^{3}$ Hsiao-Wu Hsieh, ${ }^{1}$ Kakasaheb Y. Nandiwale, ${ }^{2}$ Victor L. Schultz, ${ }^{2}$ Klavs F. Jensen, ${ }^{2}$ Richard I. Robinson*

${ }^{1}$ Global Discovery Chemistry, Novartis Institutes for Biomedical Research, 250 Massachusetts Avenue, Cambridge, MA 02139, United States

2 Department of Chemical Engineering, Massachusetts Institute of Technology, 77 Massachusetts Avenue, Cambridge, MA 02139, United States

${ }^{3}$ Chemical and Pharmaceutical Profiling, Novartis Global Drug Development, 700 Main Street South, Cambridge, MA 02139, United State

\#these authors contributed equally to the work

$\square$ kfjensen@mit.edu

$\square$ richard.robinson@novartis.com 
Table of Contents

Set-up the Self-optimizing Oscillatory Segmented Flow Reactor Platform ......................... 3

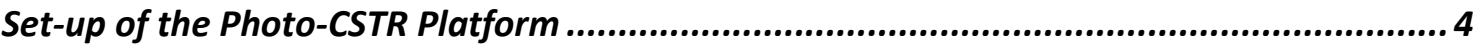

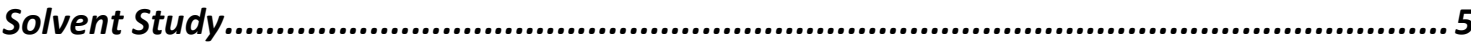

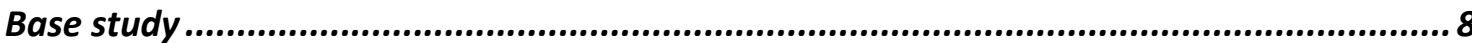

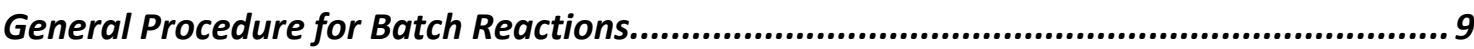

Consolidated Segmented-Flow Optimization Data........................................................... 10

General Procedure for the Experiments Using the Segmented Flow Reactor.................... 12

Methyl 4-(tetrahydro-2H-pyran-4-yl)benzoate (5).................................................................... 14

tert-butyl 4-(4-(methoxycarbonyl)benzyl)piperidine-1-carboxylate (6)................................... 15

Benzyl 4-(4-(methoxycarbonyl)benzyl)piperidine-1-carboxylate (7)......................................... 16

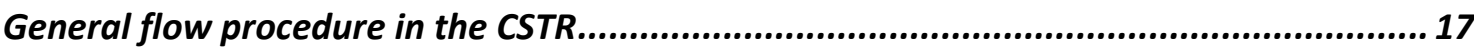

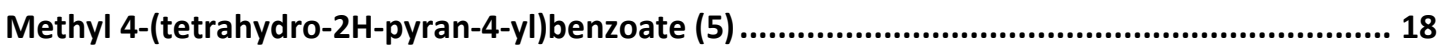

tert-Butyl 4-(4-(methoxycarbonyl)benzyl)piperidine-1-carboxylate (6)................................... 19

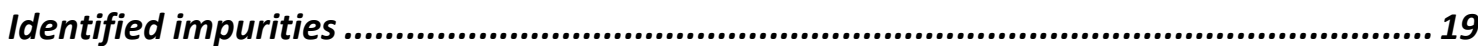

Particle Size Distributions PFR vs CSTR ..................................................................... 21 


\section{Set-up the Self-optimizing Oscillatory Segmented Flow Reactor Platform}

The fully automated oscillatory segmented flow reactor consists of a liquid handler, syringe pumps, switching valves, an oscillatory flow reactor, and an HPLC unit, is shown in Figure S1. For more information on the overall control, microslug preparation, and reactor control please refer to Hsieh et al. ${ }^{1}$
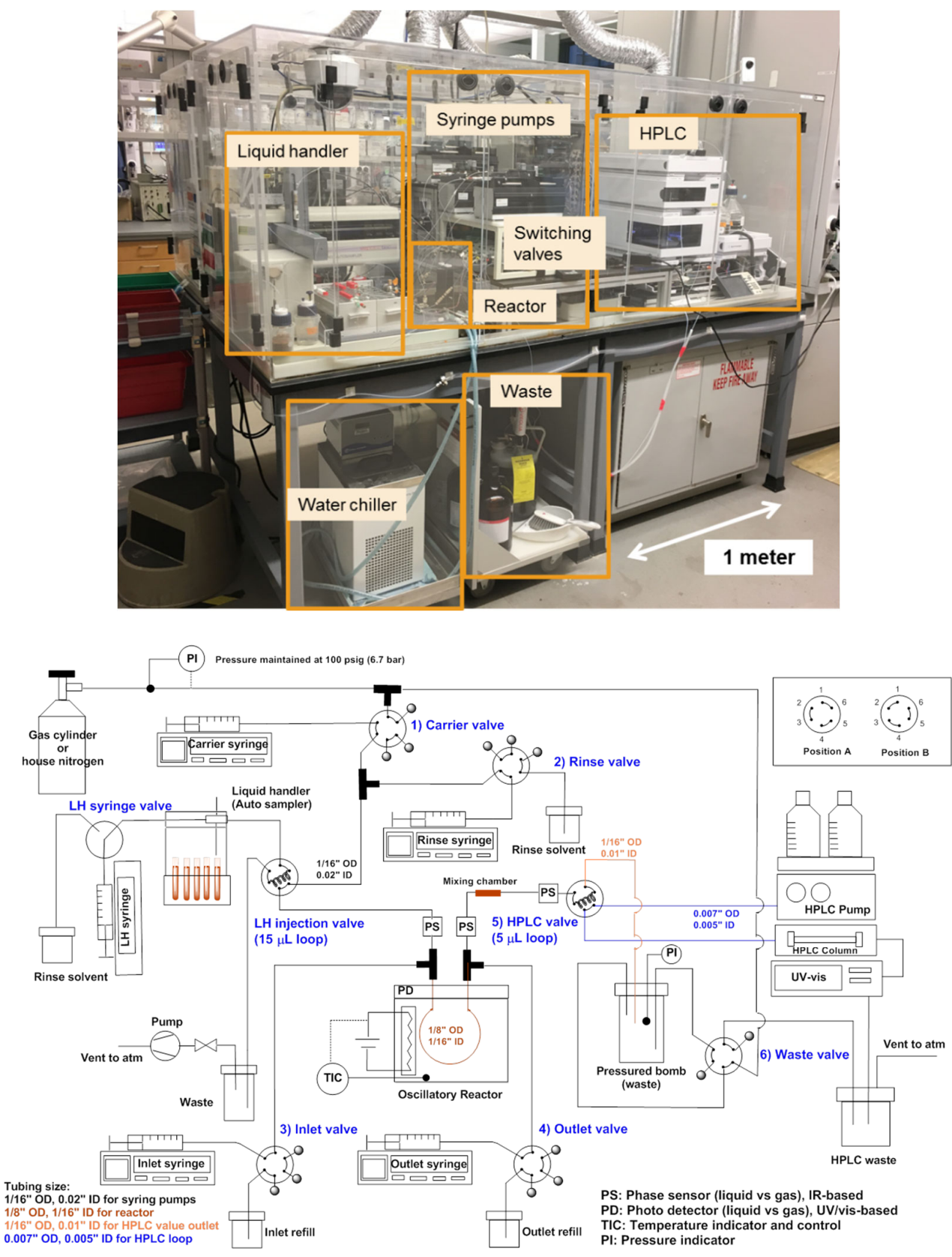

Figure S1. Image and flowchart of the automated oscillatory segmented flow reactor.

Copyright [2018] American Chemical Society. ${ }^{1}$ 


\section{Set-up of the Photo-CSTR Platform}

As shown in Figure S2, the set-up combines several syringe pumps (Harvard Model X), the CSTR cascade, blue LED light sources. Due to the strong heat formation of the LED light sources, air cooling was necessary to maintain a temperature of $35^{\circ} \mathrm{C}$ of the reactor. The reaction temperature was measured with a thermocouple wire which was squeezed in between the two aluminum covers of the CSTR. The tubes used were made of PFA with an inner diameter of $0.76 \mathrm{~mm}$. Mixing in the CSTR cascade was achieved by the magnetic stirrer (placed vertically behind the CSTR) which moves the magnetic stir bars located in the CSTR chambers (see Figure S2-S3).

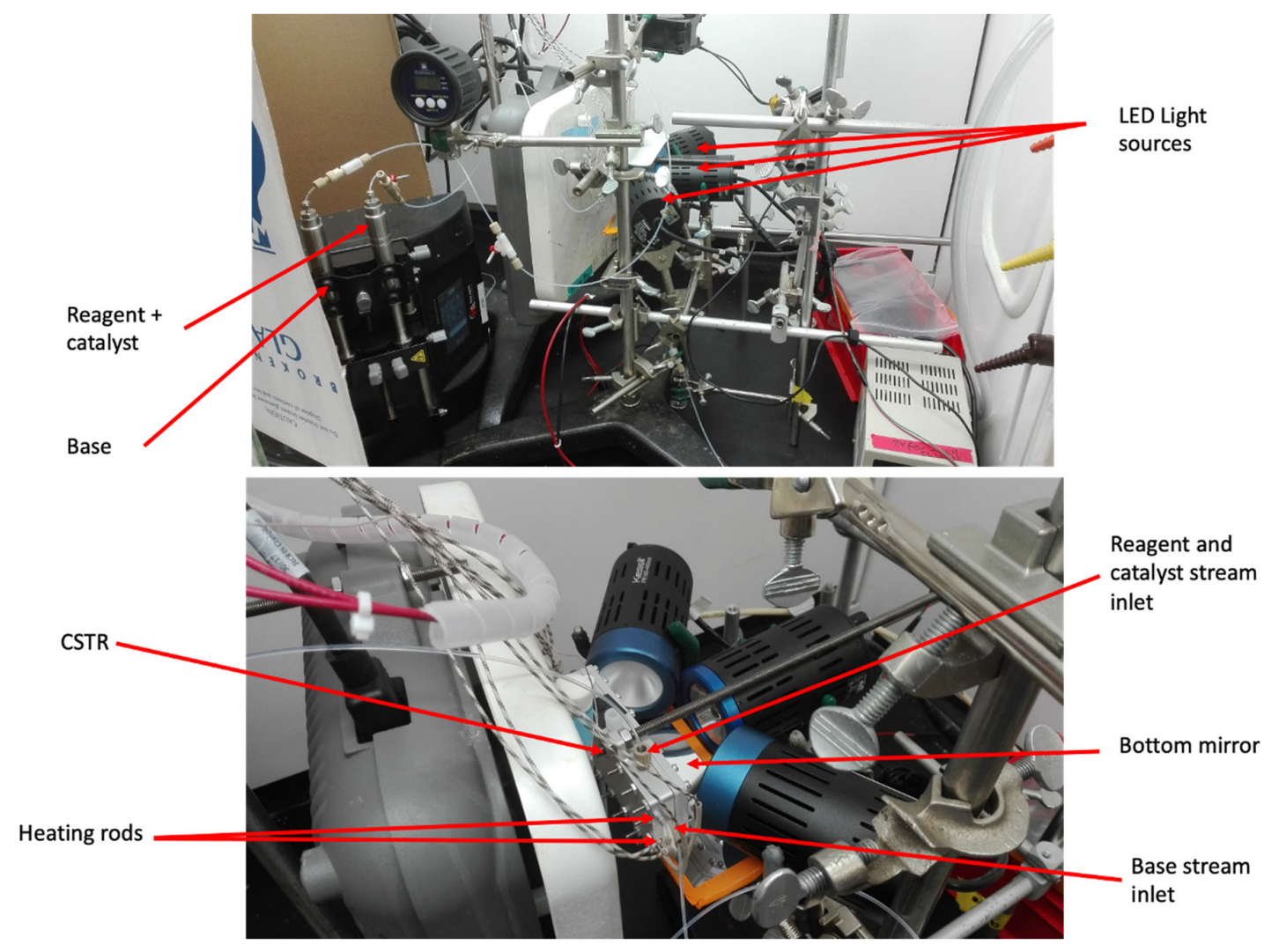

Figure S2. Experimental set-up of the photo-CSTR platform 


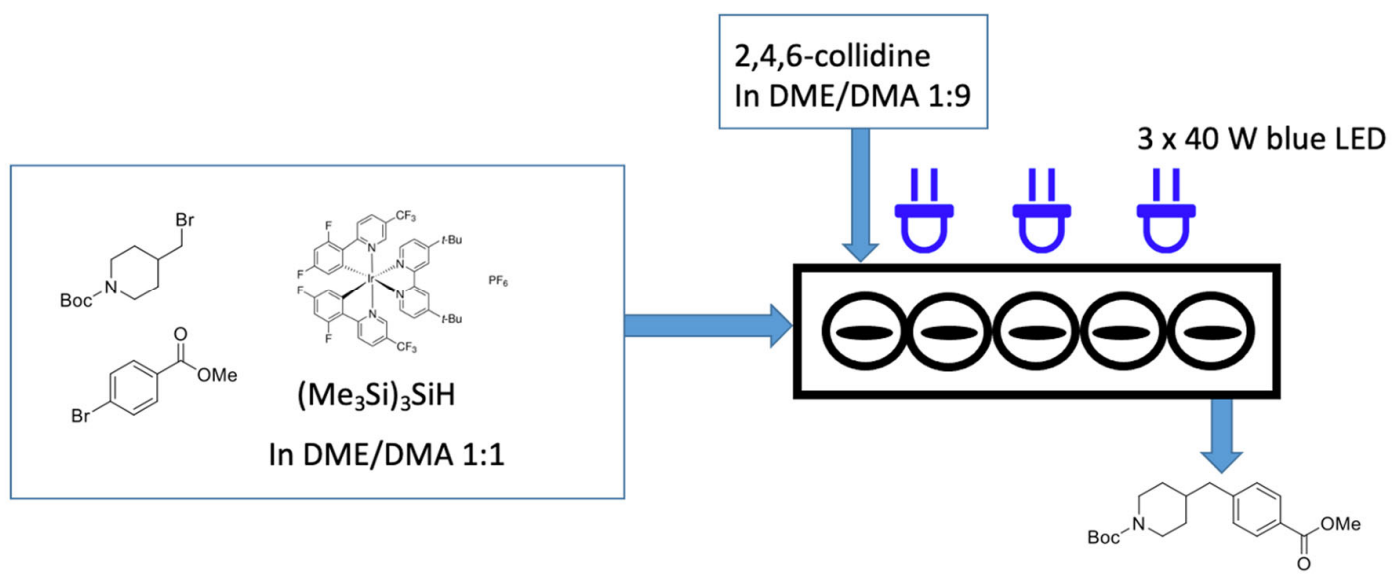

Figure S3. Flow chart of the photo-CSTR set-up

For more information on the manufacturing of the CSTR cascade please refer to Pomberger et al. $^{2}$

\section{Solvent Study}

As mentioned earlier, the ratio of 2,6-lutidine: 2,6-di-tert-butylpyridine (1:1) afforded the yield of ester 6 to $38 \%$ ( $5 \mathrm{~min}$ ) and $44 \%$ (10 $\mathrm{min}$ ) respectively at $35{ }^{\circ} \mathrm{C}$. The segmented flow reactor was then setup to screen sequential experiments, with the goal to compare the effect of 2,6lutidine and 2,6-di-tert-butylpyridine (1;1) vs. 2,6-lutidine for the cross-electrophile coupling reaction, "residence" time points $(2.5 \mathrm{~min}, 5 \mathrm{~min}, 10 \mathrm{~min}$ and $15 \mathrm{~min}$; individual automated experiments); at temperature of $35^{\circ} \mathrm{C}, 45^{\circ} \mathrm{C}$, and $55^{\circ} \mathrm{C}$ respectively as shown in Figure $\mathrm{S} 4$. We observed product degradation with extended temperature at $55^{\circ} \mathrm{C}$ in both cases. 

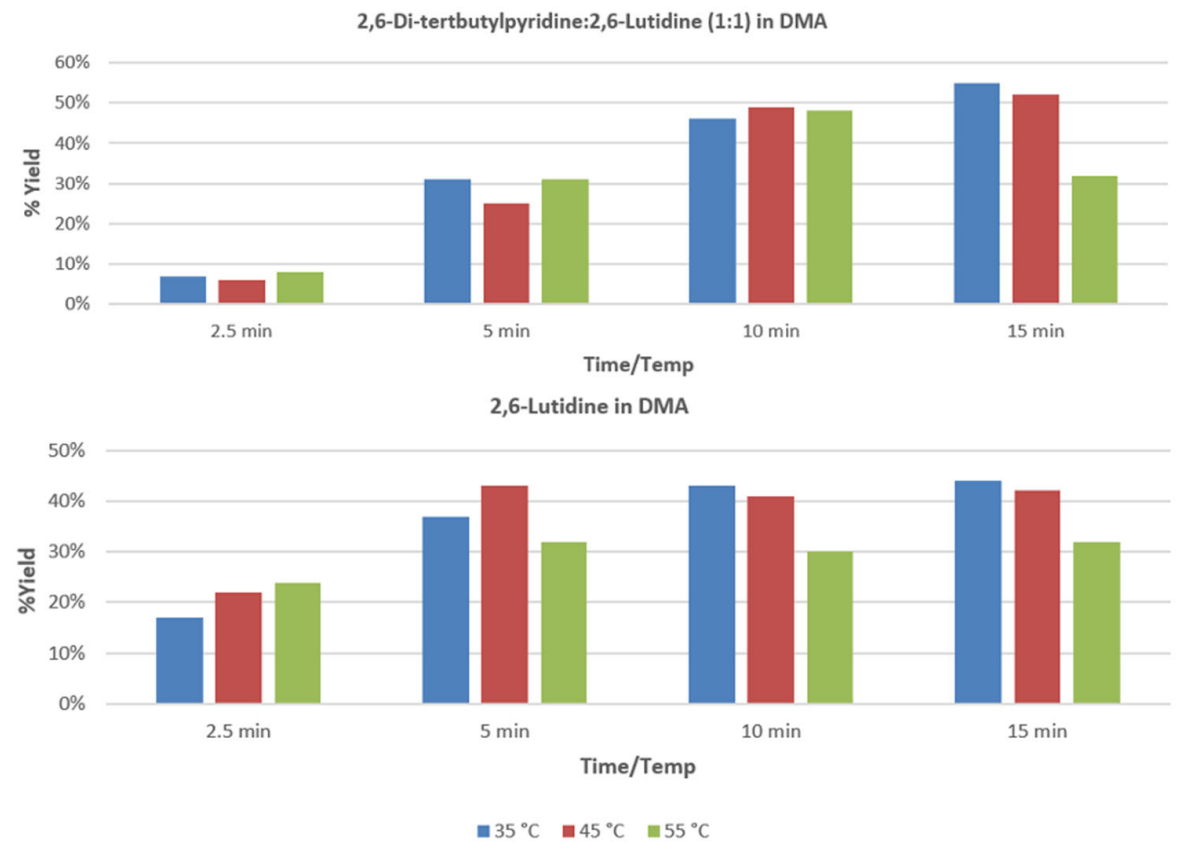

Figure S4.1 Comparison of 2,6-Di-tertbutylpridine:2,6-Lutidine (1:1) vs. 2,6-Lutidie in DMA at various time and temp

In order to further investigate the formation of the solid by-product, different reaction times and solvent ratios were screened in batch. Figure S5 illustrates the quantity of generated solids as an image (a) and within a graph (b) at different reaction times and different solvent ratios. 


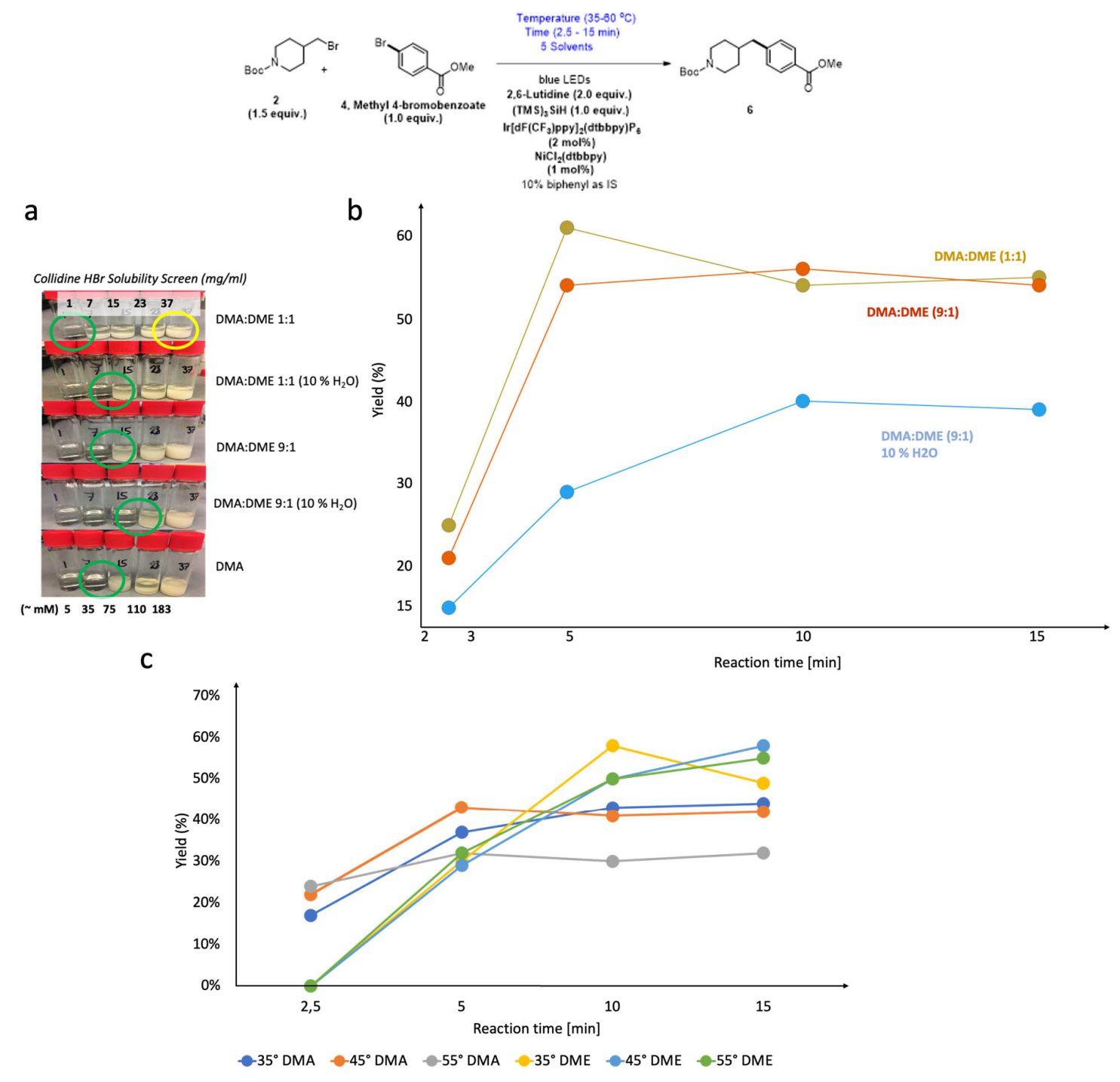

Figure S4.2. Solubility studies a) image of the reaction outcome at different solvent ratios and different reaction times b) illustration of yield at different reaction times and solvent ratios (DME:DMA mixtures using non-dried standard solvent. c) Reaction profile for DME alone and DMA at different reaction times and temperatures using 2,6-Lutidine. 


\section{Base study}

Similar to our batch experiment observation using DME as a solvent (Figure S5; 2 equivalents of pure tert-butyl pyridine), using DMA as a solvent and a high equivalence load (95\%) of ditert-butyl pyridine resulted in less than $10 \%$ of product 6 , at the 10 -minute time point with no product detectable at the 5-minute time point. Upon incremental increase of the ratio of 2,6lutidine: 2,6-di-tert-butylpyridine towards $1: 1$, the yield of ester 6 steadily increased to $38 \%$ (5 $\min )$ and $44 \%(10 \mathrm{~min})$ respectively. At the $5 \mathrm{~min}$ reaction timepoint, at ratios beyond 1.0, there was only modest improvement in the overall yield to $\mathbf{6}$, which is consistent with the recovery of the baseline reactivity (c.f. 2 equivalents of lutidine). Interestingly at the 10-minute residence time (our presumed reaction endpoint) there was a continued modest improvement in the overall yield, consistent with continued reaction. This initial data, although not conclusive seems to indicate that there is not likely a coordinative ligation effect present, which further encouraged us to pursue a pKa based pyridyl screen.

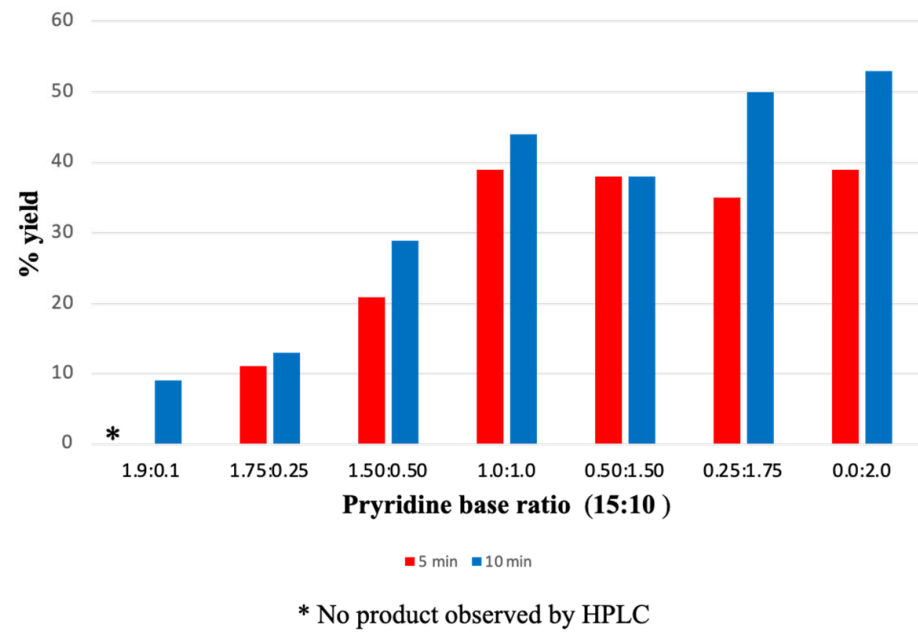

Figure S5 Using oscillatory segmented flow reactor to screen the effect of mixing 2,6-di-tertbutylpyridine with 2,6-lutidine at $35^{\circ}$ Celsius using DMA as solvent towards product 6 


\section{General Procedure for Batch Reactions}

According to Table S1, stock solutions were prepared by adding corresponding amounts of reagents to septum vials (10-mL, nitrogen flushed) and then adding anhydrous DME (0.4-5 $\mathrm{mL}$ ). Stock solutions were sonicated under nitrogen atmosphere to promote complete mixing and dissolution.

Table S1: Stock solution preparation

\begin{tabular}{|c|c|c|c|c|c|c|c|c|}
\hline Entry & Reagent & Solvent & MW & Equiv. & $\begin{array}{c}\text { Stock sol } \\
\text { conc. } \\
(\mathrm{M})\end{array}$ & $\begin{array}{c}\text { Stock } \\
\text { sol } \\
\text { volume } \\
(\mathrm{mL})\end{array}$ & $\begin{array}{c}\text { Reagent } \\
\text { mol } \\
(\mathrm{mmol})\end{array}$ & $\begin{array}{c}\text { Reagent } \\
\text { Amount } \\
(\mathrm{mg})\end{array}$ \\
\hline 1 & $\begin{array}{c}\text { Methy 4- } \\
\text { bromobezoate } \\
\text { IS: Biphenyl }\end{array}$ & $\begin{array}{c}\text { DME } \\
\text { DME }\end{array}$ & $\begin{array}{c}215.04 \\
154.21\end{array}$ & $\begin{array}{c}1.00 \\
0.10\end{array}$ & $\begin{array}{c}0.500 \\
0.050\end{array}$ & 5.00 & 2.500 & 537.6 \\
38.6 & & & & & & & & \\
\hline 2 & $\begin{array}{c}\text { 4-BrMe-N-Boc- } \\
\text { piperidine }\end{array}$ & DME & 278.19 & 1.50 & 0.750 & 3.00 & 2.250 & 625.9 \\
\hline 3 & $\begin{array}{c}\text { 4-BrMe-N-Cbz } \\
\text { piperidine }\end{array}$ & DME & 312.20 & 1.50 & 0.750 & 3.00 & 2.250 & 702.5 \\
\hline 4 & 4-bromoTHP & DME & 165.03 & 1.50 & 0.750 & 3.00 & 2.250 & 371.3 \\
\hline 5 & Ir photocatalyst & DME & 1121.91 & 0.02 & 0.010 & 5.00 & 0.050 & 56.1 \\
\hline 6 & NiCl2(dtbbpy) & DME & 398.00 & .01 & 0.005 & 5.00 & 0.025 & 10.0 \\
\hline 7 & TTMSS & DME & 248.66 & 1.00 & 0.500 & 5.00 & 2.500 & 621.7 \\
\hline 8 & Na2CO3 & DME & 105.99 & 2.00 & 1.00 & 5.00 & 5.00 & 530.0 \\
\hline 9 & 2,6-Lutidine & DME & 107.15 & 2.00 & 1.00 & 3.00 & 3.00 & 321.5 \\
\hline 10 & Barton's base & DME & 171.28 & 2.00 & 1.00 & 3.00 & 3.00 & 513.8 \\
\hline 11 & TMG & DME & 115.18 & 2.00 & 1.00 & 3.00 & 3.00 & 345.5 \\
\hline 12 & tBu-TMG & DME & 171.283 & 1.00 & 0.50 & 0.400 & 0.200 & \\
\hline 13 & $\begin{array}{c}2,2,6,6- \\
\text { tetramethylpiperidine }\end{array}$ & DME & 141.25 & 2.00 & 1.00 & 3.00 & 3.00 & 423.8 \\
\hline
\end{tabular}

To a 4-mL septum vial charged with nitrogen, reaction mixture $(2.0 \mathrm{~mL})$ was prepared by mixing DME stock solutions of methy 4-bromobezoate $(0.4 \mathrm{~mL}, 1.0$ equiv.) with $20 \mathrm{~mol} \%$ biphenyl internal standard, alkyl-bromide ( $0.4 \mathrm{~mL}, 1.5$ equiv.), $\operatorname{Ir}\left[\mathrm{dF}\left(\mathrm{CF}_{3}\right) \text { ppy }\right]_{2}(\mathrm{dtbbpy}) \mathrm{PF}_{6}$ (0.4 mL, 0.02 equiv.), $\mathrm{NiCl}_{2}$ (dtbbpy) ( $0.4 \mathrm{~mL}, 0.1$ equiv.) and base ( $0.4 \mathrm{~mL}, 1.0-2.0$ equiv.) sequentially. The reaction mixture was sealed and irradiated for 16 hours with blue LED light (H-150 lamp, $440 \mathrm{~nm}, 34 \mathrm{~W}$, Kessil Lighting) in a benchtop photochemistry reactor (EvoluChem photoredox box, HepatoChem Inc). The reaction mixture was then diluted with EtOAc $(10 \mathrm{~mL})$, washed with brine $(10 \mathrm{~mL}$ x 2), back-extracted with EtOAc $(10 \mathrm{~mL})$, dried over $\mathrm{Na}_{2} \mathrm{SO}_{4}$, filtered, and concentrated under reduced pressure to afford the crude reaction mixture as a thick oil. The resulting crude reaction mixture was then purified by either flash column chromatography (EtOAc/Heptane) or preparative $\mathrm{HPLC}\left(\mathrm{MeCN} / \mathrm{H}_{2} \mathrm{O}\right.$ with $0.1 \%$ formic acid) to afford the desired product. 


\section{Consolidated Segmented-Flow Optimization Data}

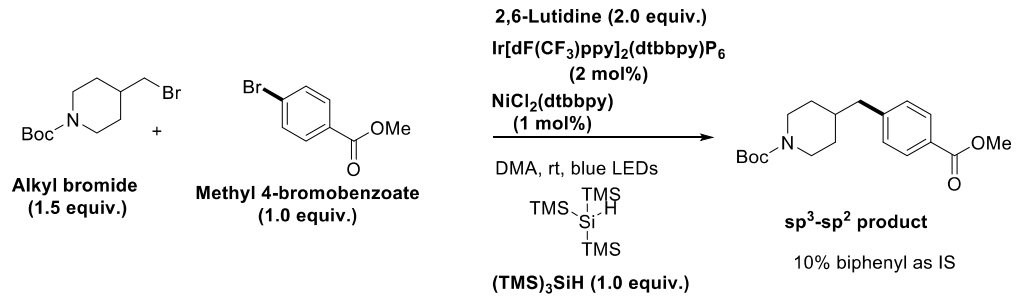

Table S2. Segmented flow reactor screening using 2,6-lutidine as base with DMA

\begin{tabular}{|c|c|c|c|c|}
\hline Entry & Temp $\left({ }^{\circ} \mathrm{C}\right)$ & Time $(\mathrm{sec})$ & Yield & \multirow{2}{*}{ Note } \\
\hline 1 & 35 & 150 & $17 \%$ & \\
\hline 2 & 35 & 300 & $37 \%$ \\
\hline 3 & 35 & 600 & $43 \%$ & \multirow{2}{*}{$100 \%$ LED } \\
\hline 4 & 35 & 900 & $44 \%$ & \\
\hline 5 & 45 & 150 & $22 \%$ & \\
\hline 6 & 45 & 300 & $43 \%$ \\
\hline 7 & 45 & 600 & $41 \%$ & \\
\hline 8 & 45 & 900 & $42 \%$ & \\
\hline 9 & 55 & 150 & $24 \%$ \\
\hline 10 & 55 & 300 & $33 \%$ \\
\hline 11 & 55 & 600 & $30 \%$ \\
\hline 12 & 55 & 900 & $32 \%$ \\
\hline
\end{tabular}

Table S3. Segmented flow reactor screening using 2,6-lutidine as base with DME:DMA $(1: 1)$

\begin{tabular}{|c|c|c|c|c|}
\hline Entry & Temp $\left({ }^{\circ} \mathrm{C}\right)$ & Time $(\mathrm{sec})$ & Yield & \multirow{2}{*}{ Note } \\
\hline 1 & 35 & 150 & $23 \%$ & \\
\hline 2 & 35 & 300 & $49 \%$ & \multirow{2}{*}{$100 \%$ LED } \\
\hline 3 & 35 & 600 & $55 \%$ & \\
\hline 4 & 35 & 900 & $69 \%$ & \\
\hline 5 & 45 & 150 & $29 \%$ & \\
\hline 6 & 45 & 300 & $56 \%$ & \\
\hline 7 & 45 & 600 & $58 \%$ & \\
\hline 8 & 45 & 900 & $63 \%$ & \\
\hline 9 & 55 & 150 & $33 \%$ & \\
\hline 10 & 55 & 300 & $51 \%$ & $52 \%$ \\
\hline 11 & 55 & 600 & 900 & $54 \%$ \\
\hline 12 & 55 & & & \\
\hline
\end{tabular}




\begin{tabular}{|l|l|l|l|}
\hline 13 & 47.5 & 300 & $62 \%$ \\
\hline 14 & 47.5 & 150 & $35 \%$ \\
\hline 15 & 47.5 & 300 & $59 \%$ \\
\hline 16 & 47.5 & 600 & $66 \%$ \\
\hline
\end{tabular}

Table S4. Segmented flow reactor screening using 2,4,6-collidine as base with DME:DMA (1:1)

\begin{tabular}{|c|c|c|c|c|}
\hline Entry & Temp $\left({ }^{\circ} \mathrm{C}\right)$ & Time (sec) & Yield & Note \\
\hline 1 & 35 & 150 & $25 \%$ & \multirow{16}{*}{$100 \%$ LED } \\
\hline 2 & 35 & 300 & $61 \%$ & \\
\hline 3 & 35 & 600 & $54 \%$ & \\
\hline 4 & 35 & 900 & $55 \%$ & \\
\hline 5 & 45 & 150 & $28 \%$ & \\
\hline 6 & 45 & 300 & $59 \%$ & \\
\hline 7 & 45 & 600 & $57 \%$ & \\
\hline 8 & 45 & 900 & $59 \%$ & \\
\hline 9 & 55 & 150 & $30 \%$ & \\
\hline 10 & 55 & 300 & $56 \%$ & \\
\hline 11 & 55 & 600 & $55 \%$ & \\
\hline 12 & 55 & 900 & - & \\
\hline 13 & 37.5 & 300 & $64 \%$ & \\
\hline 14 & 37.5 & 375 & $64 \%$ & \\
\hline 15 & 35 & 420 & $59 \%$ & \\
\hline 16 & 37.5 & 420 & $42 \%$, & \\
\hline
\end{tabular}




\section{General Procedure for the Experiments Using the Segmented Flow Reactor}<smiles>COC(=O)c1ccc(C2CCCCC2)cc1</smiles>

The stock solution was prepared according to Table S1. Then, small quantities of the stock solutions $(0.5-1.0 \mathrm{~mL})$ were transferred to empty dried and nitrogen-flushed 4-mL septum vials in the liquid handler vial rack. Stock solutions were freshly prepared prior the experiment and replaced daily to ensure the best experimental outcomes.

\section{Quantification using HPLC}

For more information on calculation and procedure on quantification using HPLC, please refer to Hsieh et al. ${ }^{1}$ All details on the HPLC analysis can be found on Figures S6a-c.

\begin{tabular}{|c|c|c|c|c|c|c|c|c|c|c|c|c|}
\hline $\begin{array}{l}\text { Sample } \\
\text { No. }\end{array}$ & $\begin{array}{c}\text { [Produrt] } \\
\text { (M) }\end{array}$ & $\begin{array}{c}\text { Volume } \\
\text { (mL) }\end{array}$ & $\begin{array}{l}\text { Product } \\
\text { (mmol) }\end{array}$ & {$[\mid \mathrm{S}](\mathrm{M})$} & $\begin{array}{l}\text { Volume } \\
(\mathrm{mL})\end{array}$ & Is (mmol) & $\begin{array}{c}\text { Total } \\
\text { volume } \\
(\mathrm{mL})\end{array}$ & $\begin{array}{l}\text { Final }[P] \\
\text { (M) }\end{array}$ & $\begin{array}{l}\text { Final [IS] } \\
(\mathrm{M})\end{array}$ & [PY|S] & 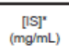 & 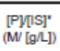 \\
\hline s1 & 0.05 & 0.20 & 0.01 & 0.05 & 0.020 & 0.00100 & 0.22 & 0.04545 & 0.005 & 10.00 & 0.701 & 0.08485 \\
\hline & 0.05 & & & 0.05 & & & & & & & & \\
\hline s3 & 0.05 & 0.20 & 0.01 & 0.05 & 0.080 & 0.00400 & 0.280 & 0.03571 & 0.014 & 2.50 & 2.203 & $\begin{array}{l}0.01621 \\
0.01621\end{array}$ \\
\hline$\$ 4$ & 0.05 & 0.20 & 0.01 & 0.05 & 0.200 & 0.01000 & 0.400 & 0.02500 & 0.025 & 1.00 & 3.865 & 0.00848 \\
\hline s5 & 0.05 & 0.20 & 0.01 & 0.05 & 0.400 & 0.02000 & 0.600 & 0.01867 & 0.033 & 0.50 & 5.140 & 0.00324 \\
\hline se & 0.05 & 0.20 & 0.01 & 0.06 & 0.600 & 0.02500 & 0.700 & $0.0142 \theta$ & 0.036 & 0.40 & 5.508 & 0.00250 \\
\hline $\begin{array}{l}\mathrm{S7} \\
\mathrm{s} 8 \\
\end{array}$ & $\begin{array}{l}0.05 \\
0.05\end{array}$ & $\begin{array}{l}0.20 \\
0.20\end{array}$ & 0.01 & 0.05 & $\begin{array}{l}0.800 \\
2000\end{array}$ & $\begin{array}{l}0.04000 \\
0.10000\end{array}$ & $\begin{array}{l}1.000 \\
2200\end{array}$ & $\begin{array}{l}0.01000 \\
0.00455\end{array}$ & 0.040 & $\begin{array}{l}0.25 \\
0.10\end{array}$ & $\begin{array}{l}0.168 \\
7010\end{array}$ & $\begin{array}{l}0.001622 \\
0.00085\end{array}$ \\
\hline & & & & & & & & & & & & \\
\hline $\begin{array}{c}\text { Product } \\
(\mathrm{mg})\end{array}$ & Product & Produot & $0.05 \mathrm{M}$ & & IS (mg) & IS MN & IS (mmol) & $0.05 \mathrm{M}$ & & & & \\
\hline & $\frac{M N}{220}$ & $\frac{(\mathrm{mmol})}{0.150}$ & $\frac{5 t 00 k \mathrm{~mL}}{3.00}$ & & 77.105 & 154.21 & 0.5 & $\frac{5 \text { stook } \mathrm{mL}}{10}$ & & & & \\
\hline
\end{tabular}
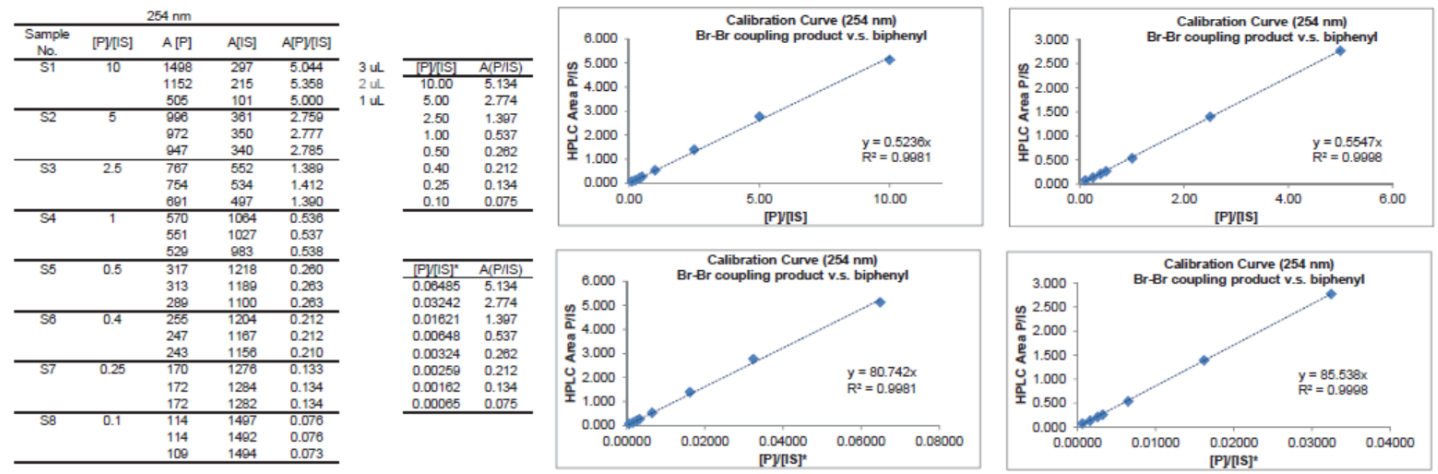

Figure S6.a Compound 5 vs biphenyl: Calculation table, HPLC results, and calibration curves 


\begin{tabular}{|c|c|c|c|c|c|c|c|c|c|c|c|c|}
\hline $\begin{array}{c}\text { Sample } \\
\text { No. }\end{array}$ & $\begin{array}{l}\text { [Product] } \\
\text { (M) }\end{array}$ & $\begin{array}{c}\text { Volume } \\
(\mathrm{mL})\end{array}$ & $\begin{array}{l}\text { Product } \\
\text { (mmol) }\end{array}$ & {$[\mathrm{S}](\mathrm{M})$} & $\begin{array}{c}\text { Volume } \\
(\mathrm{mL})\end{array}$ & IS (mmol) & $\begin{array}{c}\text { Total } \\
\text { volume } \\
(\mathrm{mL})\end{array}$ & $\begin{array}{c}\text { Final [P] } \\
\text { (M) }\end{array}$ & $\begin{array}{c}\text { Final [IS] } \\
\text { (M) }\end{array}$ & [PYIS] & $\underset{(\mathrm{mg} / \mathrm{mL})}{[\mathrm{IS}]^{*}}$ & 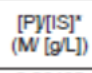 \\
\hline S1 & 0.1 & 0.15 & 0.015 & 0.1 & 0.015 & 0.002 & 0.165 & 0.091 & 0.009 & 10.00 & 1.402 & 0.06485 \\
\hline S2 & 0.1 & 0.15 & 0.015 & 0.1 & 0.030 & 0.003 & 0.180 & 0.083 & 0.017 & 5.00 & 2.570 & 0.03242 \\
\hline s3 & 0.1 & 0.15 & 0.015 & 0.1 & 0.060 & 0.006 & 0.210 & 0.071 & 0.029 & 2.50 & 4.406 & 0.01621 \\
\hline S4 & 0.1 & 0.15 & 0.015 & 0.1 & 0.150 & 0.015 & 0.300 & 0.050 & 0.050 & 1.00 & 7.711 & 0.00648 \\
\hline S5 & 0.1 & 0.15 & 0.015 & 0.1 & 0.300 & 0.030 & 0.450 & 0.033 & 0.087 & 0.50 & 10.281 & 0.00324 \\
\hline 56 & 0.1 & 0.15 & 0.015 & 0.1 & 0.600 & 0.060 & 0.750 & 0.020 & 0.080 & 0.25 & 12.337 & 0.00162 \\
\hline s7 & 0.1 & 0.15 & 0.015 & 0.1 & 0.750 & 0.075 & 0.900 & 0.017 & 0.083 & 0.20 & 12.851 & 0.00130 \\
\hline S8 & 0.1 & 0.15 & 0.015 & 0.1 & 1.000 & 0.100 & 1.150 & 0.013 & 0.087 & 0.15 & 13.410 & 0.00097 \\
\hline $\begin{array}{l}\text { Product } \\
\text { (mg) }\end{array}$ & $\begin{array}{c}\text { Product } \\
\text { MW }\end{array}$ & $\begin{array}{l}\text { Product } \\
(\mathrm{mmol})\end{array}$ & $\begin{array}{c}0.1 \mathrm{M} \\
\text { stock } \mathrm{mL}\end{array}$ & & IS (mg) & IS MW & IS (mmol) & $\begin{array}{c}0.1 \mathrm{M} \\
\text { stock } \mathrm{mL}\end{array}$ & & & & \\
\hline 31 & 232 & 0.134 & 1.34 & & 154 & 154.21 & 1 & 10 & & & & \\
\hline
\end{tabular}

\begin{tabular}{ccccc}
\multicolumn{5}{c}{$254 \mathrm{~nm}$} \\
\hline $\begin{array}{ccccc}\text { Sample } \\
\text { No. }\end{array}$ & [PYIS] & A [P] & A[IS] & A[PY[IS] \\
\hline S1 & 10 & 2504 & 370 & 6.768 \\
& & 2424 & 367 & 6.605 \\
& & 2406 & 359 & 6.702 \\
\hline S2 & 5 & 4521 & 1247 & 3.628 \\
& & 4456 & 1228 & 3.629 \\
& & 2242 & 628 & 3.570 \\
\hline S3 & 2.5 & 3300 & 1777 & 1.857 \\
& & 3339 & 1801 & 1.854 \\
& & 1676 & 839 & 1.998 \\
\hline S4 & 1 & 2238 & 2872 & 0.779 \\
& & 2239 & 2867 & 0.781 \\
& & 1174 & 1487 & 0.790 \\
\hline S5 & 0.5 & 1259 & 3255 & 0.387 \\
& & 1248 & 3227 & 0.387 \\
& & 685 & 1638 & 0.406 \\
\hline S6 & 0.25 & 771 & 3683 & 0.209 \\
& & 431 & 1895 & 0.227 \\
& & 433 & 1901 & 0.228 \\
\hline S7 & 0.2 & 393 & 2150 & 0.183 \\
& & 397 & 2175 & 0.183 \\
& & 397 & 2172 & 0.183 \\
\hline S8 & 0.15 & 324 & 2186 & 0.148 \\
& & 324 & 2183 & 0.148 \\
& & 323 & 2167 & 0.149 \\
\hline
\end{tabular}
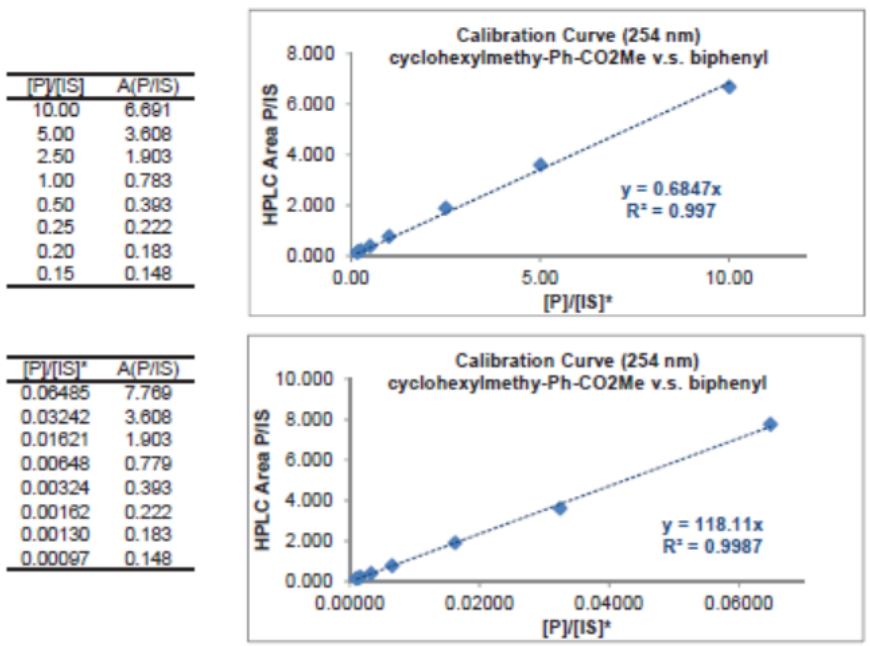

Figure S6.b Compound 6 vs biphenyl: Calculation table, HPLC results, and calibration curves
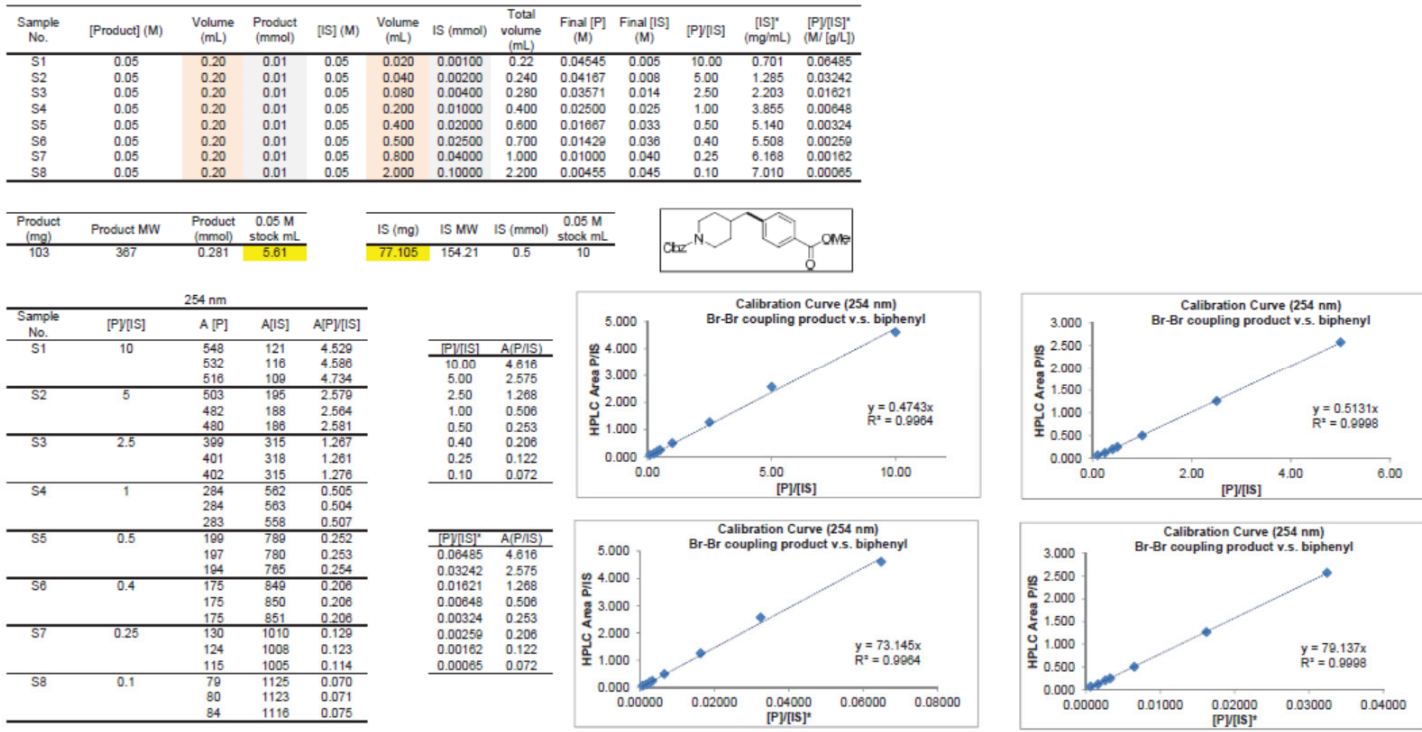

Figure S6.c Compound 7 vs biphenyl: Calculation table, HPLC results, and calibration curves 
Methyl 4-(tetrahydro-2H-pyran-4-yl)benzoate (5).

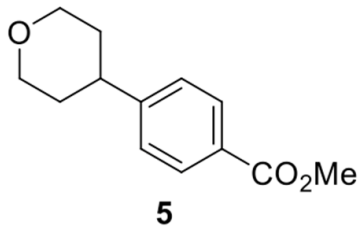

The compound was synthesized from methy 4-bromobezoate (4) and 4-bromo-THP (1) according to the general procedure for segmented flow reactor.

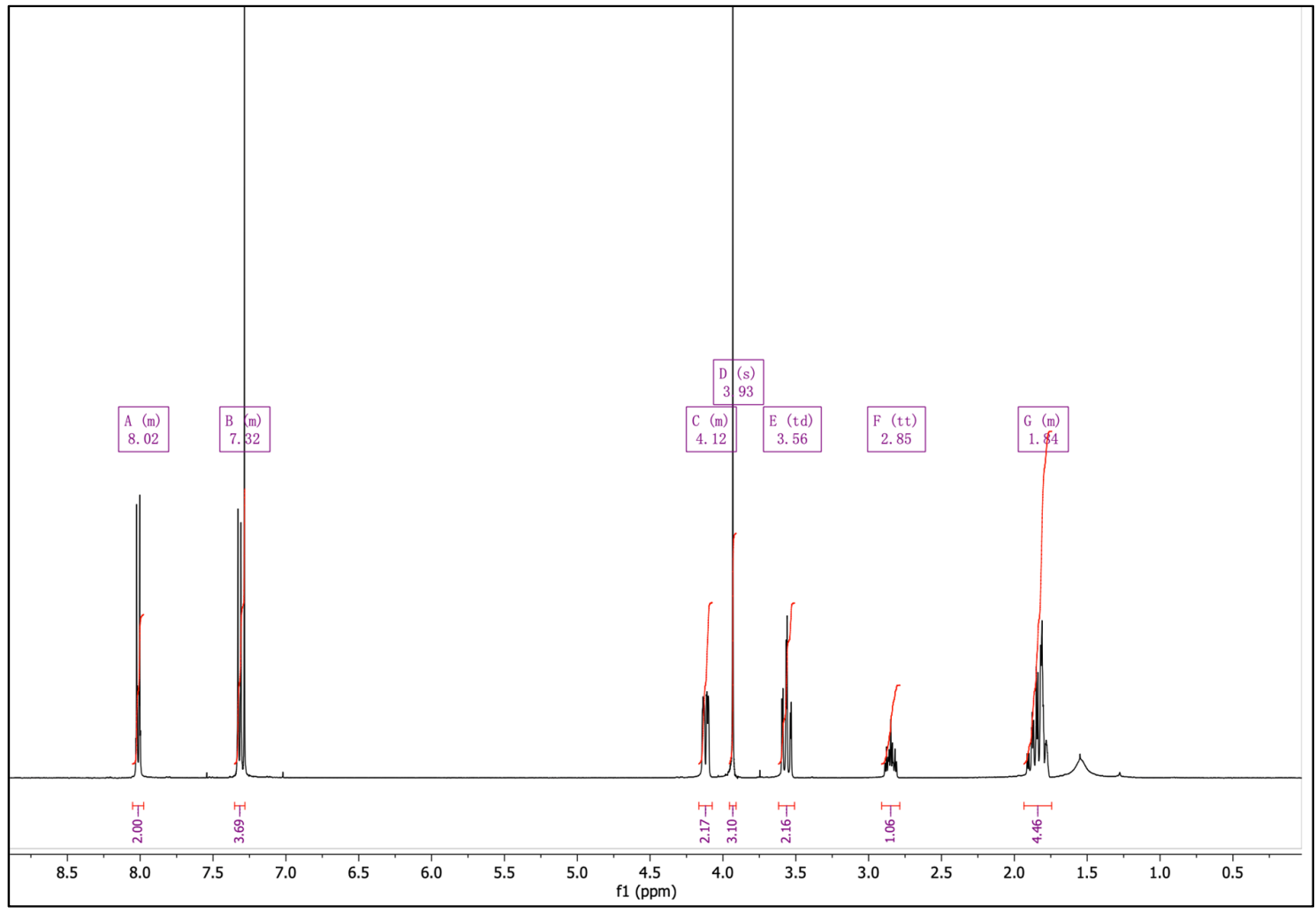

${ }^{1} \mathrm{H}$ NMR (400 MHz, Chloroform-d) $\delta 8.03$ - 7.96 (m, 2H), $7.34-7.28$ (m, 2H), 4.14 - 4.06 (m, 2H), 3.91 (s, 3H), 3.54 (td, J = 11.5, $2.6 \mathrm{~Hz}, 2 \mathrm{H}), 2.83$ (tt, J = 11.5, $4.4 \mathrm{~Hz}, 1 \mathrm{H}), 1.92-$ $1.77(\mathrm{~m}, 4 \mathrm{H})$.

${ }^{13} \mathrm{C}$ NMR (101 MHz, Chloroform-d) $\delta 167.2,151.1,129.8,128.3,126.8,68.2,52.1,41.8,33.56$ 
tert-butyl 4-(4-(methoxycarbonyl)benzyl)piperidine-1-carboxylate (6).<smiles>CC(=O)c1ccc(CC2CCN(C(=O)OC(C)(C)C)CC2)cc1</smiles>

The compound was synthesized from methyl 4-bromobezoate (4) and 4-bromo-Me-N-Bocpiperidine (2) according to the general procedure for segmented flow reactor.

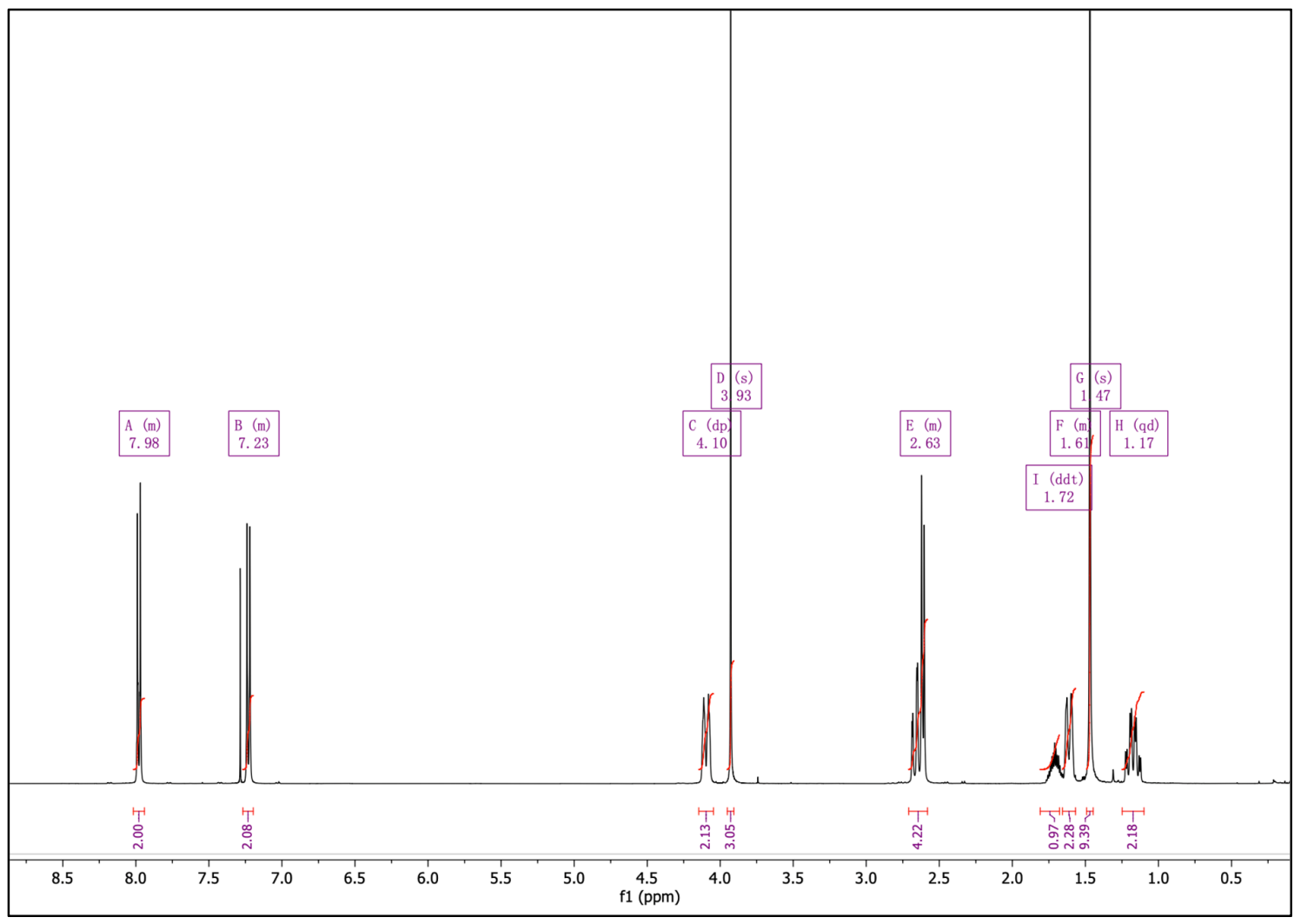

${ }^{1} \mathrm{H}$ NMR (400 MHz, Chloroform-d) $\delta 8.00$ - 7.89 (m, 2H), 7.25 - 7.15 (m, 2H), 4.08 (d, J = $13.4 \mathrm{~Hz}, 2 \mathrm{H}), 3.91(\mathrm{~s}, 3 \mathrm{H}), 2.71-2.54(\mathrm{~m}, 4 \mathrm{H}), 1.70$ (th, $\mathrm{J}=11.0,3.6 \mathrm{~Hz}, 1 \mathrm{H}), 1.63-1.52(\mathrm{~m}$, 2H), 1.45 (s, 9H), $1.24-1.08(\mathrm{~m}, 2 \mathrm{H})$.

${ }^{13} \mathrm{C}$ NMR (101 MHz, CDCl3) $\delta 167.1,154.8,145.8,129.6,129.14,128.0,79.3,52.0,43.9$, $43.1,38.0,32.0,28.5$.

HRMS (ESI): $\mathrm{m} / \mathrm{z}$ [M+Na]+ calculated for C19H27NO4Na 356.1837, found: 356.1843 
Benzyl 4-(4-(methoxycarbonyl)benzyl)piperidine-1-carboxylate (7)<smiles>COC(=O)c1ccc(CC2CCN(C(=O)OCc3ccccc3)CC2)cc1</smiles>

The compound was synthesized from methyl 4-bromobezoate (4) and 4-Br-Me-N-Cbz piperidine (3) according to the general procedure for segmented flow reactor.

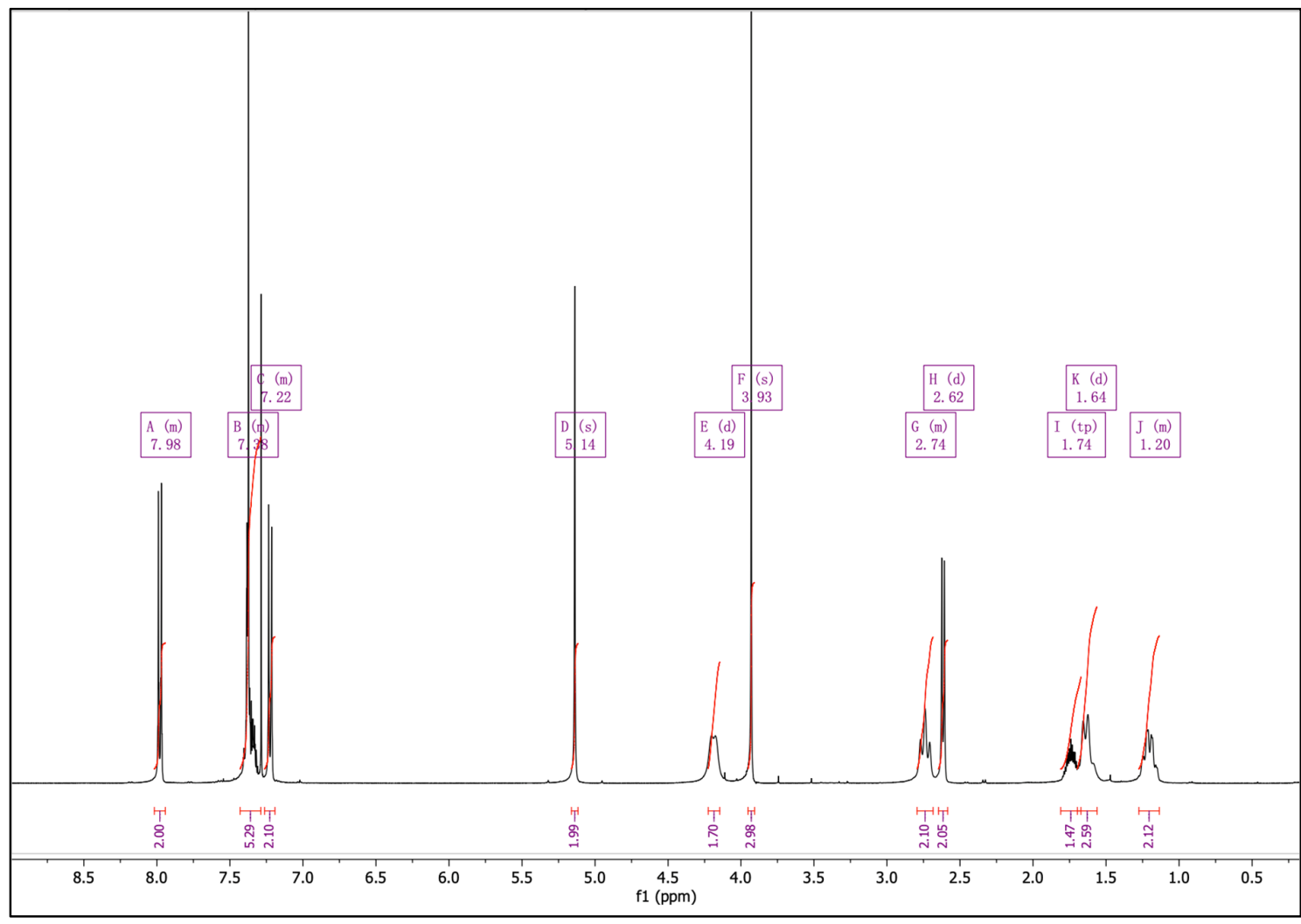

${ }^{1} \mathrm{H}$ NMR (400 MHz, Chloroform- $d$ ) $\delta 8.02-7.94$ (m, 2H), 7.43 - 7.29 (m, 5H), 7.26 - 7.19 $(\mathrm{m}, 2 \mathrm{H}), 5.14(\mathrm{~s}, 2 \mathrm{H}), 4.19(\mathrm{~d}, J=13.0 \mathrm{~Hz}, 3 \mathrm{H}), 3.93(\mathrm{~s}, 3 \mathrm{H}), 2.79-2.68(\mathrm{~m}, 2 \mathrm{H}), 2.62(\mathrm{~d}, J=$ $7.1 \mathrm{~Hz}, 2 \mathrm{H}), 1.64(\mathrm{~d}, J=13.6 \mathrm{~Hz}, 3 \mathrm{H}), 1.20(\mathrm{qd}, J=12.5,4.3 \mathrm{~Hz}, 2 \mathrm{H})$.

${ }^{13} \mathrm{C}$ NMR (101 MHz, Chloroform- $d$ ) $\delta 167.1,155.3,145.7,137.0,129.7,129.2,128.5,128.1$, $128.0,127.9,67.0,52.1,44.1,43.1,37.9,31.9$. 


\section{General flow procedure in the CSTR}

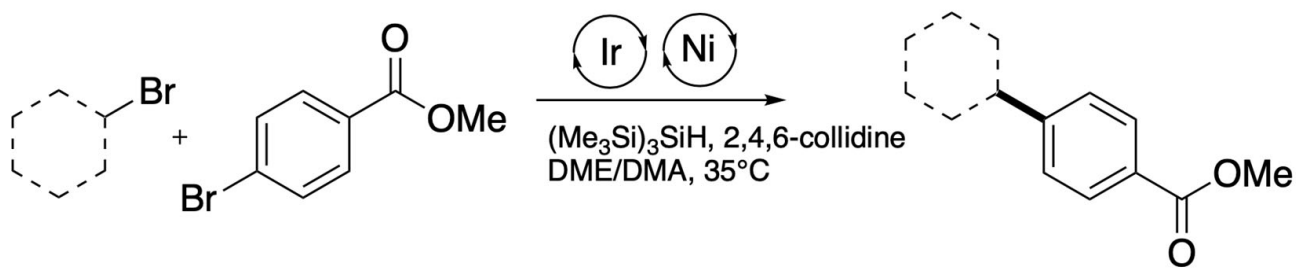

Methyl 4-bromobenzoate (1 equiv., $230 \mathrm{mmol} / \mathrm{L})$, alkylbromide (1.5 equiv., $345 \mathrm{mmol} / \mathrm{L}$ ), $\operatorname{Ir}[\mathrm{dF}(\mathrm{CF} 3)$ ppy]2(dtbbpy)PF6 (2 mol\%), NiCl2(dtbbpy) (1 mol\%), (Me3Si)3SiH (1.3 equiv.), naphthalene (10 mol\%, internal HPLC standard) and 1,3,5- trimethoxybenzene (10 mol\%, internal NMR standard) were added to a closed dry vial. A solvent combination (DMA:DME, 1:1 degassed by sparging with helium) was added and degassed for 10 more minutes (in the vial) by sparging with helium. The mixture was sonicated until it became homogeneous and then transferred to a steel syringe. To a closed dry vial containing 2,4,6-collidine $(800 \mathrm{mmol} / \mathrm{L})$ a solvent mixture (DMA:DME, 9:1, degassed by sparging with helium) was added and then transferred in another steel syringe. The CSTR was purged with dry argon and then filled with degassed DME. Both syringes were placed in a Harvard syringe pump and connected to the CSTR. The flow rate for both syringe pumps was set to $20 \mu \mathrm{L} / \mathrm{min}$ (2.25 hours residence time), stirring in the CSTR was started, irradiation was started ( $3 \mathrm{x}$ blue LED $440 \mathrm{~nm}, 40 \mathrm{Watt})$ and air cooling was started. In order to stabilize the system temperature at $35-37^{\circ} \mathrm{C}$ the CSTR was simultaneously heated (via the heating rods) and cooled with air. After 6.75 hours of continuous operation (three residence times) the product outflow was collected in a vial for 60 minutes (2400 $\mu \mathrm{L}$ were collected). For work-up, the suspension was washed with aq. $\mathrm{K}_{2} \mathrm{CO}_{3}$ solution, the organic phase was reextracted with ethyl acetate, dried over $\mathrm{MgSO}_{4}$ and then evaporated under vacuum. The reaction yield and conversion were quantified with LC-MS and confirmed with quantitative ${ }^{1} \mathrm{H}$ NMR. Purification in order to analyze the pure product via NMR was performed via preparative HPLC and is described in detail below. 


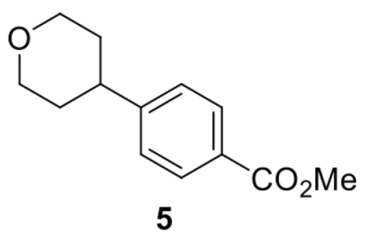

Following general flow procedure, using 4-bromotetrahydropyran (1.5 equiv, $345 \mathrm{mmol} / \mathrm{L}, 57$ $\mathrm{mg} / \mathrm{mL}$ ) and methyl 4-bromobenzoate (1 equiv., $230 \mathrm{mmol} / \mathrm{L}, 50 \mathrm{mg} / \mathrm{mL}$ ). The collected sample (collection during 60 minutes, worked-up, extracted, dried and evaporated) was purified by preparative $\mathrm{HPLC}\left(\mathrm{MeCN} / \mathrm{H}_{2} \mathrm{O}\right.$ with $0.1 \%$ formic acid) to afford the product as a colorless oil.

LC-MS: 70\% yield, 93\% conversion.

Isolated yield product 5: $39 \mathrm{mg}, 0.177 \mathrm{mmol}, 65 \%$.

During 60 minutes of sampling, $1.2 \mathrm{~mL}$ of the substrate stream containing methyl 4bromobenzoate (1 equiv., $230 \mathrm{mmol} / \mathrm{L}, 0.276 \mathrm{mmol}$ ) was pumped into the system.

${ }^{1} \mathrm{H}$ and ${ }^{13} \mathrm{C}$ NMR matches previously reported data from the segmented flow experiments. 
tert-Butyl 4-(4-(methoxycarbonyl)benzyl)piperidine-1-carboxylate (6)

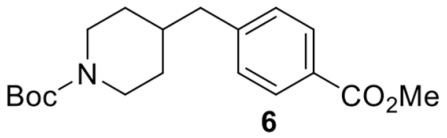

Following general flow procedure A, using tert-butyl 4-(bromomethyl)piperidine-1carboxylate (1.5 equiv, $345 \mathrm{mmol} / \mathrm{L})$ and methyl 4-bromobenzoate (1 equiv., $230 \mathrm{mmol} / \mathrm{L})$. The collected sample (collection during 60 minutes, worked-up, extracted, dried and evaporated) was purified by preparative HPLC (MeCN/ $\mathrm{H} 2 \mathrm{O}$ with $0.1 \%$ formic acid) to afford the product as colorless oil.

LC-MS: $62 \%$ yield $74 \%$ conversion.

Isolated yield product 9: $52 \mathrm{mg}, 0.156 \mathrm{mmol}, 57 \%$.

During 60 minutes of sampling, $1.2 \mathrm{~mL}$ of the substrate stream containing methyl 4bromobenzoate ( 1 equiv., $230 \mathrm{mmol} / \mathrm{L}, 0.276 \mathrm{mmol}$ ) was pumped into the system. During the 24 hours steady-state operation experiment the whole outflow was collected and purified by column chromatography. Yield was identical to the shorter experiments.

${ }^{1} \mathrm{H}$ and ${ }^{13} \mathrm{C}$ NMR matches previously reported data from the segmented flow experiments.

\section{Identified impurities}

Based on isolated ${ }^{1} \mathrm{H}$ NMR we propose the following impurity to be generated during the reaction when using DME as system solvent.

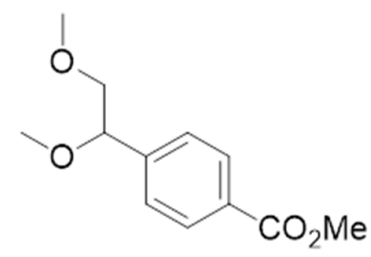

1H NMR (400 MHz, CDCl3) $\delta 7.92-7.83(\mathrm{~m}, 2 \mathrm{H}), 7.30-7.22(\mathrm{~m}, 2 \mathrm{H}), 4.28(\mathrm{dd}, \mathrm{J}=7.8,3.6$ $\mathrm{Hz}, 1 \mathrm{H}), 3.76(\mathrm{~s}, 3 \mathrm{H}), 3.43(\mathrm{dd}, \mathrm{J}=10.4,7.7 \mathrm{~Hz}, 1 \mathrm{H}), 3.32-3.21(\mathrm{~m}, 4 \mathrm{H}), 3.15(\mathrm{~s}, 3 \mathrm{H})$ 


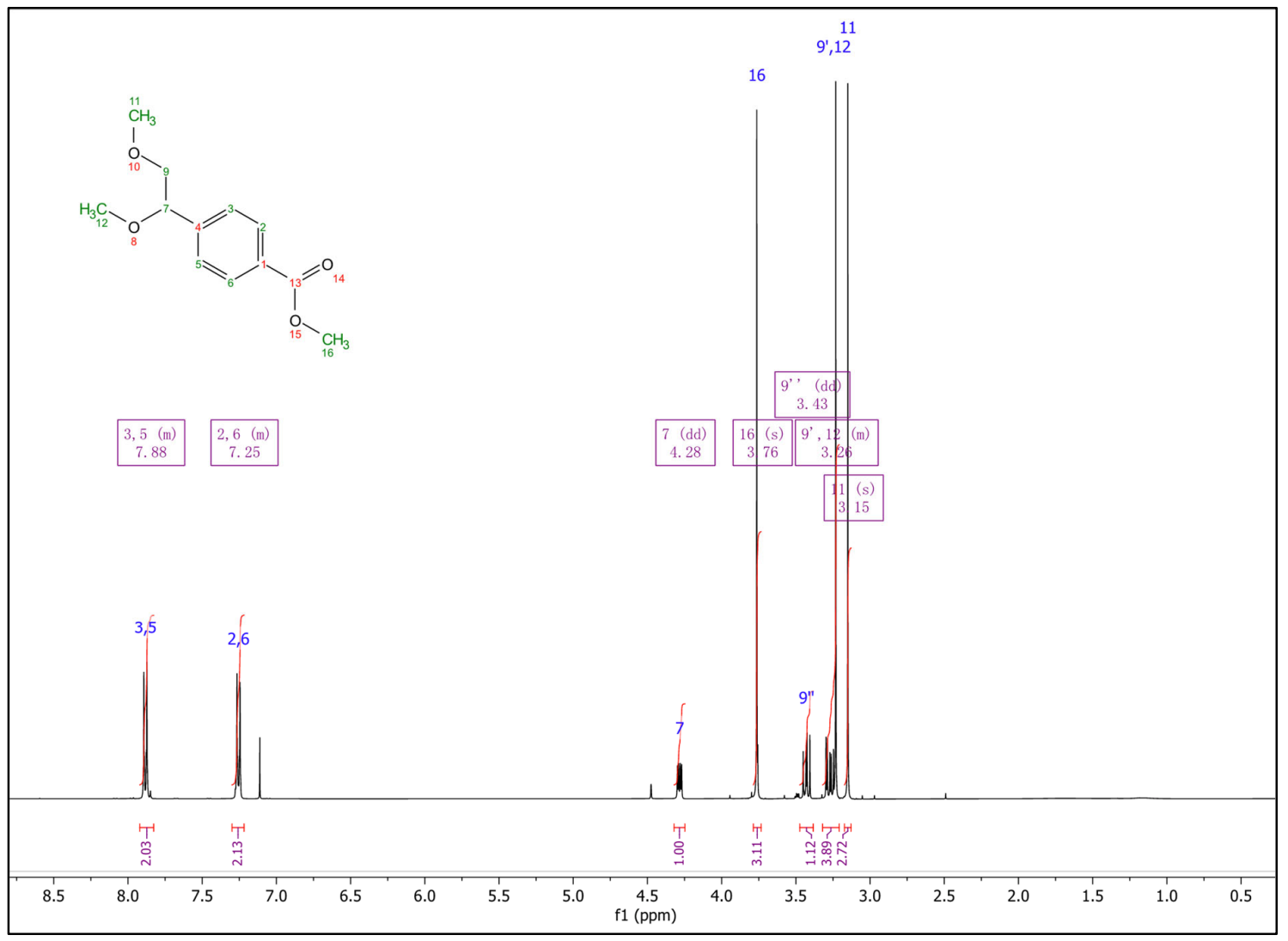

We postulate the solvent related impurities are generated through a background $\boldsymbol{C}$ - $\boldsymbol{H}$ radical abstraction process, with the potential to generate competing $1^{\circ}$ or $2^{\circ}$ solvent alkyl radicals. These solvent derived radicals can accumulate and intercept the Nickel catalytic cycle ${ }^{3}$ (competing against the desired $1^{\circ}$ alkyl substrate radical species - green)-Figure S7.

Figure S7: Proposed mechanistic rationale for observed DME solvent related background reaction.

In the case of our reaction we exclusively observe the DME adduct, derived exclusively from the $2^{\circ}$ alkyl radical species. The $2^{\circ}$ DME radical should be inherently more stable than its $1^{\circ}$ 
counterpart with potential for both to be generated. However, due to the increased stability of the oxygen stabilized $2^{\circ}$ radical, as a consequence it likely has enough longevity (and hence concentration build up) to enable it to compete against the primary $1^{\circ}$ Halogen Radical abstraction pathway of the substrate. Hence consideration of solvent selection is of significance when designing these reaction processes to minimize undesirable background reactions.

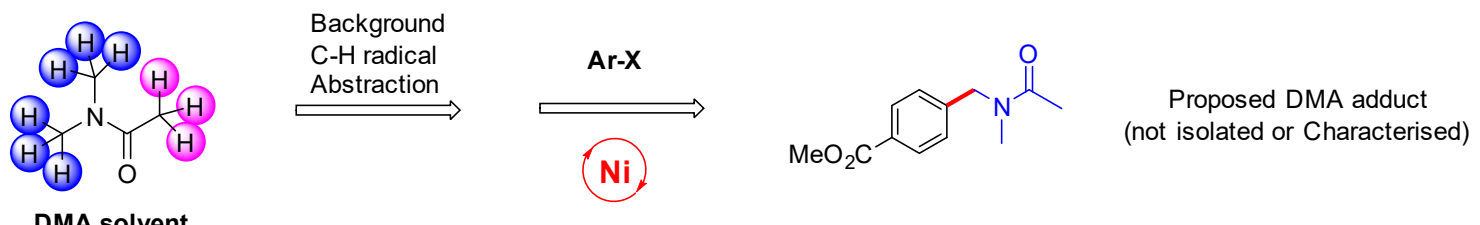

Figure S8: Proposed DMA adduct based on MS observation.

\section{Particle Size Distributions PFR vs CSTR}

We initially postulated that the CSTR (due the mechanical agitation) may result in differing particle size distribution verses the PFR reactor, which conversely could allow uninhibited crystal growth in solution during the reaction progression. Samples were taken during the course of extended $24 \mathrm{hr}$ CSTR run and the PSD (Particle Size Distrbution) was taken to compare to the PFR (prior to blockage).

Scanning electron microscopy (SEM) imaging of the collidine- $\mathrm{HBr}$ particles obtained in DMA:DME (9:1) revealed formation of crystalline microrods in the PFR (Figure S9a).

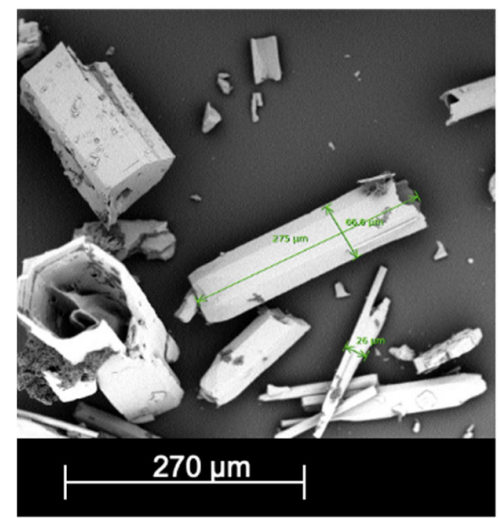

Figure S9a: SEM micrographs of colldine-HBr microrod crystals in Vapourtec UV-150 10 $\mathrm{mL}$ reactors 


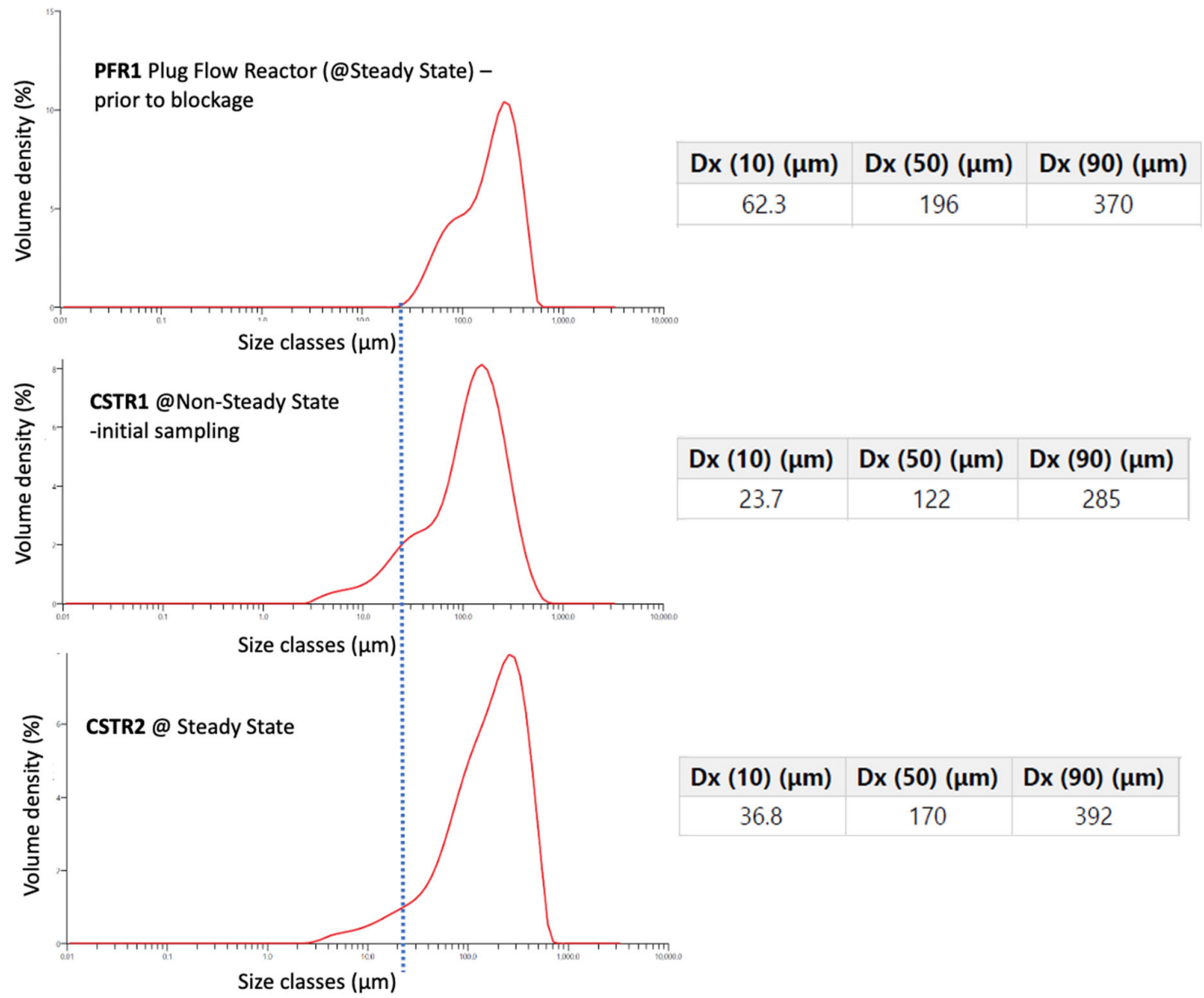

Figure S9b: PSD distributions PFR vs CSTR (initial and steady state sampling)

Although indeed we did observe a greater extent of smaller crystals [shown by average distribution at D10 (\%) PFR1 (63) vs CSTR1 (23) / CSTR2 (37)], in the CSTR vs the PFR, indicating some particle size reduction, there was still an observable increase in particle size by approx $40 \%$ from the inital CSTR1 (collected during 3-3.5h of operation) timepoint to CSTR 2 (collected during 8.5-9.75h of operation) when taking into account D10-D90 entire distributions (Figure S9b).

Overall, there was not a significant difference in the overall PSD at steady state between the two reactors (Figure S9c) 


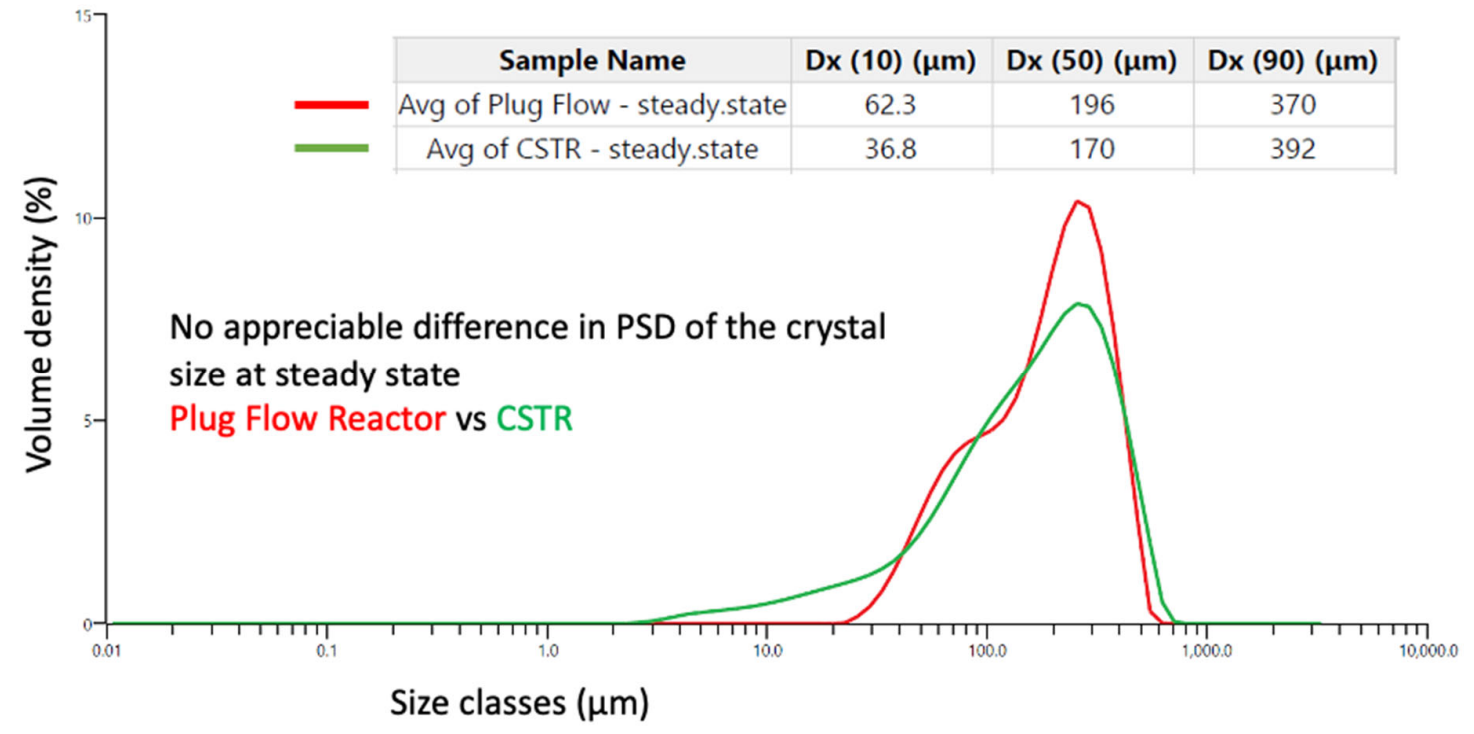

Figure S9c: Overlay of particle size distribution on aliquots of mixtures exiting reactor at steady state (PFR vs CSTR). 
References

1. Hsieh, H.-W.; Coley, C. W.; Baumgartner, L. M.; Jensen, K. F.; Robinson, R. I., Photoredox Iridium-Nickel Dual-Catalyzed Decarboxylative Arylation Cross-Coupling: From Batch to Continuous Flow via Self-Optimizing Segmented Flow Reactor. Organic Process Research \& Development 2018, 22 (4), 542-550.

2. Pomberger, A.; Mo, Y.; Nandiwale, K. Y.; Schultz, V. L.; Duvadie, R.; Robinson, R. I.; Altinoglu, E. I.; Jensen, K. F., A Continuous Stirred-Tank Reactor (CSTR) Cascade for Handling Solid-Containing Photochemical Reactions. Organic Process Research \& Development 2019, 23 (12), 2699-2706.

3. See Figure 2; Patricia, Zhang, Chi "Chip" Le, and David W. C. MacMillan Silyl Radical Activation of Alkyl Halides in Metallaphotoredox Catalysis: A Unique Pathway for Cross-Electrophile Coupling; Journal of the American Chemical Society 2016138 (26), 8084-8087 